\title{
NORMING NIL ALGEBRAS
}

\author{
H. G. DALES
}

ABSTRACT. A commutative nil algebra with countable basis is normable, but a commutative nilpotent algebra is not necessarily normable.

Let $A$ be a commutative algebra over the complex field, C. We say that $A$ is normable if there is an algebra norm on $A$, or, equivalently, if $A$ can be embedded in a normed algebra. A deep recent theorem of Esterle [3] shows that, if the continuum hypothesis holds, then each integral domain without identity, of cardinality at most that of the continuum is normable. For a discussion of this result, and of related results, see $[2, \S 9]$. We consider here an opposite extreme, the normability of nil algebras.

Throughout, we consider only commutative, linear associative algebras over $\mathbf{C}$. Let $A$ be an algebra. If $n \in \mathrm{N}$, we write $A^{n}$ for the linear span of the set of products of $n$ elements of $A$. An element $a$ of $A$ is nil if $a^{n}=0$ for some $n \in \mathbf{N}$, the algebra $A$ is nilpotent if $A^{n}=0$ for some $n \in \mathrm{N}$, and $A$ is nil if each element of $A$ is nilpotent. A vector space norm, $\|$.$\| , on an algebra A$ is submultiplicative, or an algebra norm, if $\|a b\| \leqslant\|a\|\|b\|(a, b \in A)$.

First, suppose that $A$ is a nilpotent algebra.

If $A^{2}=0$, then certainly $A$ is normable. For let $\left\{a_{\alpha}\right\}$ be a basis for $A$ over $\mathbf{C}$, and set $\left\|\Sigma_{1}^{n} \lambda_{i} a_{\alpha_{i}}\right\|=\Sigma_{1}^{n}\left|\lambda_{i}\right|$. Then $\|$.$\| is a vector space norm on A$, and in this case each vector space norm is an algebra norm.

Now consider the case that $A^{3}=0$. An example to show that such an $A$ may not be normable is already implicit in the note [4] of Esterle. If $p>1$, let $l^{p}$ denote the usual Banach space. Then $l^{p}$ is a commutative Banach algebra with respect to coordinatewise multiplication, and $l^{q}$ is an ideal in $l^{p}$ if $1<q<p$. Let $A=l^{p} / l^{q}$. Then, by [4, Theorem 3.1], $A$ is normable if and only if $p<2 q$. By taking $p, q$ so that $3 q \geqslant p>2 q \geqslant 2$, we obtain an algebra $A$ with $A^{3}=0$ which is not normable. However, this example uses the "main boundedness theorem" of Bade and Curtis [1], and it may be of interest to give a totally elementary example.

EXAMPLE 1. There exists a commutative algebra $A$ with $A^{3}=0$ which is not normable.

Proof. As a vector space, $A$ has as basis the set $\left\{e_{s}: s \in[0,1)\right\}$. The multiplication is given by

$$
e_{s} e_{t}= \begin{cases}e_{0} /|s-t| & (s, t \in(0,1), s \neq t), \\ 0 & \text { (otherwise) }\end{cases}
$$

Received by the editors June 12, 1980.

AMS (MOS) subject classifications (1970). Primary 46J05; Secondary 16A22.

Key words and phrases. Normed algebra, nil algebra, nilpotent algebra.

(C) 1981 American Mathematical Society 0002-9939/81/0000-0416/\$02.00 
It is clear that the product of any three elements of $A$ is zero, so that $A$ is associative and $A^{3}=0$.

Now suppose that $\|\cdot\|$ is an algebra norm on $A$. Then

$$
0<\left\|e_{0}\right\| \leqslant|s-t|\left\|e_{s}\right\|\left\|e_{t}\right\| \quad(s, t \in(0,1), s \neq t) .
$$

For $n \in \mathbf{N}$, let $U_{n}=\left\{s \in(0,1):\left\|e_{s}\right\|>n\right\}$. If $s \in(0,1)$ and if $0<|t-s|<$ $\left\|e_{0}\right\| / n^{2}\left\|e_{s}\right\|$, then $t \in U_{n}$. Thus, each $U_{n}$ is open and dense in $(0,1)$. By Baire's theorem, $\cap U_{n} \neq \varnothing$, a contradiction. Thus $A$ is not normable.

The above example clearly uses the uncountability of the dimension of $A$. In fact, we shall now show that each nil, and in particular each nilpotent, algebra of countable dimension is normable. (A proof, shorter than the following, that each nilpotent algebra of countable dimension is normable can easily be given.) The result is an easy consequence of the following lemma.

LEMMA 2. Let $B$ be a finite-dimensional algebra, and let $A$ be a nil subalgebra such that $B$ is algebraically generated by $A$ and by an element $b_{0}$ of $B$ with $b_{0}^{2} \in A$. Suppose that there is an algebra norm on $A$. Then there is an algebra norm on $B$ extending the norm on $A$.

Proof. Let $a_{1}, \ldots, a_{p}$ be a basis of $A$. Then $\left\{b_{0}+A, a_{1} b_{0}+A, \ldots, a_{p} b_{0}+A\right\}$ spans the vector space $B / A$. Choose a linearly independent subset of this set, say it is $\left\{z_{1}+A, \ldots, z_{k}+A\right\}$, where $z_{1}, \ldots, z_{k} \in B$. Then each $b \in B$ has a unique expression in the form $b=a+\sum_{1}^{n} \alpha_{i} z_{i}$, with $a \in A$ and $\alpha_{1}, \ldots, \alpha_{k} \in C$ : we set $\pi(b)=\left(\alpha_{1}, \ldots, \alpha_{k}\right)$.

Note that

$$
z_{i} z_{j} \in A \quad(i, j=1, \ldots, k)
$$

because $\left(a_{r} b_{0}\right)\left(a_{s} b_{0}\right) \in A$ for $r, s=1, \ldots, p$.

For $a \in A$, let $T_{a}: \mathbf{C}^{k} \rightarrow \mathbf{C}^{k}$ be defined by

$$
T_{a}\left(\lambda_{1}, \ldots, \lambda_{k}\right)=\pi\left[a\left(\lambda_{1} z_{1}+\cdots+\lambda_{k} z_{k}\right)\right] .
$$

Then $T_{a}$ is a linear map on $C^{k}$, and we regard $T_{a}$ as an element of $M_{k}(C)$. The map $a \mapsto T_{a}$ of $A$ into $M_{k}(C)$ is an algebra homomorphism, and so $\left\{T_{a}: a \in A\right\}$ is a set of commuting matrices in $M_{k}(C)$. Thus, by [5, p. 134], we can choose a basis of $C^{k}$ so that each matrix $T_{a}$ is lower triangular with respect to the new basis. By replacing each $z_{i}$ by the appropriate linear combination of $z_{1}, \ldots, z_{k}$, we can suppose that each $T_{a}$ already has a lower triangular matrix. Note that equation (1) still holds for the new $z_{1}, \ldots, z_{k}$. Let $T_{a}=\left[m_{i j}(a)\right]$ for $a \in A$, where each $m_{i j}$ is a linear functional on $A$ and $m_{i j}=0$ if $j>i$. Clearly, each $m_{i i}$ is a homomorphism $A \rightarrow \mathbf{C}$.

Take $a \in A$ and let $\phi: A \rightarrow \mathbf{C}$ be a homomorphism. If $\phi(a) \neq 0$, let $b=\phi(a)^{-1} a$. Since $A$ is a nil algebra, there exists $c \in A$ with $b+c=b c$, so that $1+\phi(c)=$ $\phi(c)$, a contradiction. Thus $\phi(a)=0$. This shows that we can suppose that $T_{a}=$ [ $\left.m_{i j}(a)\right]$ for $a \in A$, where each $m_{i j}$ is a linear functional on $A$ and $m_{i j}=0$ if $j>i$.

For $j \in\{1, \ldots, k\}$, let $L_{j}=\operatorname{lin}\left\{A, z_{1}, \ldots, z_{j}\right\}$, and take $L_{0}=A$. Then we have shown that, with our choice of $z_{1}, \ldots, z_{k}$, we have $A z_{j} \subset L_{j-1}(j=1, \ldots, k)$. 
Using (1), it follows that

$$
B z_{j} \subset L_{j-1} \quad(j=1, \ldots, k) .
$$

We shall successively define $\left\|z_{1}\right\|, \ldots,\left\|z_{k}\right\|$ (with $\left\|z_{j}\right\|>0$ ). When we have defined $\left\|z_{1}\right\|, \ldots,\left\|z_{j}\right\|$ we extend the norm to $L_{j}$ by setting

$$
\left\|a+\alpha_{1} z_{1}+\cdots+\alpha_{j} z_{j}\right\|=\|a\|+\left|\alpha_{1}\right|\left\|z_{1}\right\|+\cdots+\left|\alpha_{j}\right|\left\|z_{j}\right\| .
$$

Clearly, we obtain on each $L_{j}$ a norm which extends the norm on $A$. We shall show that, with our choice of the $\left\|z_{j}\right\|$, the norm on $L_{k}=B$ is submultiplicative.

In fact, we choose $\left\|z_{i}\right\|$ so that for $i=1, \ldots, k$,

$$
\left\|a z_{i}\right\| \leqslant\|a\|\left\|z_{i}\right\| \quad\left(a \in L_{i-1}\right) \text { and }\left\|z_{i}^{2}\right\|<\left\|z_{i}\right\|^{2} .
$$

Suppose that $j=1$, or that $j>1$ and that $\left\|z_{1}\right\|, \ldots,\left\|z_{j-1}\right\|$ have been chosen so that (3) holds for $i=1, \ldots, j-1$. If $a \in L_{j-1}$, then $a z_{j} \in L_{j-1}$ by (2), and so $\left\|a z_{j}\right\|$ and $\left\|z_{j}^{2}\right\|$ have already been defined. Define a norm on $L_{j-1}^{2}$ by setting $\|(x, y)\|=\|x\|+\|y\|$, and let $X_{j}=\left\{(a, b) \in L_{j-1}^{2}: a z_{j}=b\right\}$. Then $X_{j}$ is a linear subspace of the finite-dimensional normed space $L_{j-1}^{2}$, and hence $X_{j}$ is closed. Let $Y_{j}=\left\{(a, b) \in X_{j}:\|b\|=1\right\}$, a closed subset of $L_{j-1}^{2}$. The map $(a, b) \mapsto\|a\|$ is a continuous function on $L_{j-1}^{2}$, and clearly $\delta_{j} \equiv \inf \left\{\|a\|:(a, b) \in Y_{j}\right\}$ is attained. If $(a, b) \in Y_{j}$, then $a \neq 0$, and so $\delta_{j}>0$. We take $\left\|z_{j}\right\|=\max \left\{\delta_{j}^{-1},\left\|z_{j}^{2}\right\|^{1 / 2}\right\}$, and it is then easy to check that (3) holds in the case that $i=j$. Thus, we can choose $\left\|z_{1}\right\|, \ldots,\left\|z_{k}\right\|$ so that (3) holds for $i=1, \ldots, k$.

We now confirm that the norm is indeed submultiplicative on $B$. Take $a, b \in B$, say $a=a_{0}+\sum_{1}^{k} \alpha_{i} z_{i}, \quad b=b_{0}+\sum_{1}^{k} \beta_{i} z_{i}$, where $a_{0}, b_{0} \in A$, and $\alpha_{1}, \ldots, \alpha_{k}$, $\beta_{1}, \ldots, \beta_{k} \in \mathrm{C}$. Then $a b=a_{0} b_{0}+\sum_{1}^{k} c_{j} z_{j}+\Sigma_{1}^{k} \alpha_{j} \beta_{j} z_{j}^{2}$, where

$$
c_{j}=\alpha_{j} b_{0}+\beta_{j} a_{0}+\sum_{i=1}^{j-1}\left(\alpha_{i} \beta_{j}+\alpha_{j} \beta_{i}\right) z_{i},
$$

so that $c_{j} \in L_{j-1}$. Then

$$
\|a b\| \leqslant\left\|a_{0} b_{0}\right\|+\sum_{1}^{k}\left\|c_{j} z_{j}\right\|+\sum_{1}^{k}\left|\alpha_{j} \beta_{j}\right|\left\|z_{j}^{2}\right\| .
$$

But $\left\|a_{0} b_{0}\right\| \leqslant\left\|a_{0}\right\|\left\|b_{0}\right\|$ because $\|\cdot\|$ is an algebra norm on $A$, and $\left\|c_{j} z_{j}\right\|<$ $\left\|c_{j}\right\|\left\|z_{j}\right\|,\left\|z_{j}^{2}\right\| \leqslant\left\|z_{j}\right\|^{2}$ by (3). Thus

$$
\begin{aligned}
\|a b\| \leqslant & \left\|a_{0}\right\|\left\|b_{0}\right\|+\left\|b_{0}\right\| \sum\left|\alpha_{j}\right|\left\|z_{j}\right\|+\left\|a_{0}\right\| \sum\left|\beta_{j}\right|\left\|z_{0}\right\| \\
& +\sum_{i=1}^{j-1}\left(\left|\alpha_{i}\right|\left|\beta_{j}\right|+\left|\alpha_{j}\right|\left|\beta_{i}\right|\right)\left\|z_{i}\right\|\left\|z_{j}\right\|+\sum_{1}^{k}\left|\alpha_{j}\right|\left|\beta_{j}\right|\left\|z_{j}\right\|^{2} \\
\leqslant & \left(\left\|a_{0}\right\|+\sum\left|\alpha_{j}\right|\left\|z_{j}\right\|\right)\left(\left\|b_{0}\right\|+\sum\left|\beta_{j}\right|\left\|z_{j}\right\|\right) \\
= & \|a\|\|b\|,
\end{aligned}
$$

as required.

We now give our result.

TheORem 3. Let $A$ be a nil algebra with countable basis. Then $A$ is normable. 
Proof. We can write $A=\cup_{n=0}^{\infty} A_{n}$, where $A_{0}=\{0\}$, where each $A_{n}$ is a finite-dimensional subalgebra of $A_{n+1}$, and where $A_{n+1}$ is the algebra generated by $A_{n}$ and by an element $a_{n+1}$ of $A_{n+1}$ with $a_{n+1}^{2} \in A_{n}$. The algebras $A_{n}$ are nil, and so we can successively extend an algebra norm from $A_{n}$ to $A_{n+1}$. Hence, we can construct an algebra norm on $\boldsymbol{A}$.

A result which is apparently more general is also true.

TheOREM 4. Let $A$ be a radical algebra with countable basis. Then $A$ is normable.

However, the extra generality is illusory because a theorem of Amitsur (see [6, p. 20]) shows that a radical algebra with countable basis is, in fact, a nil algebra.

It is not true that every algebra with countable basis can be normed.

EXAMPLE 5. There exists a commutative algebra $A$ with a countable basis which is not normable.

Proof. Let $P$ be the free polynomial algebra in the countable family of variables $\left(X_{n}: n \in \mathrm{N}\right.$ ), and let $P_{0}$ denote the polynomials with zero constant term. Let $I$ be the ideal in $P_{0}$ generated by the elements $X_{1} X_{n}-n X_{n}(n>2)$, and let $A=P_{0} / I$. Then $A$ is a commutative algebra with a countable basis, and we shall show that $A$ is not normable.

First note that $X_{n} \notin I$ for $n \geqslant 2$. For if so, there exists $n>2, k \in \mathbf{N}$, and $p_{2}, \ldots, p_{k} \in P_{0}$ with $X_{n}=\sum_{j=2}^{k}\left(X_{1} X_{j}-j X_{j}\right) p_{j}$. Take $X_{1}=X, X_{n}=Y$, and $X_{j}=0$ for $j \in \mathbf{N} \backslash\{1, n\}$. Then $Y=(X Y-n Y) p(X, Y)$ for a polynomial $p$, and so $1=(X-n 1) p(X, Y)$, which is not possible. Thus $X_{n} \notin I$ for $n>2$, as required.

Suppose that $\|\cdot\|$ is an algebra norm on $A$. Set $a_{n}=X_{n}+I \in A$ for $n \in \mathbf{N}$. Then $a_{1} a_{n}=n a_{n}(n \geqslant 2)$, and so $n\left\|a_{n}\right\| \leqslant\left\|a_{1}\right\|\left\|a_{n}\right\|(n>2)$. Since $X_{n} \notin I,\left\|a_{n}\right\| \neq$ 0 for $n \geqslant 2$. Thus $n \leqslant\left\|a_{1}\right\|(n \geqslant 2)$, a contradiction. Hence $A$ is not normable.

\section{REFERENCES}

1. W. G. Bade and P. C. Curtis, Homomorphisms of commutative Banach algebras, Amer. J. Math. 82 (1960), 589-608.

2. H. G. Dales, Automatic continuity: a survey, Bull. London Math. Soc. 10 (1978), 129-183.

3. J. Esterle, Homomorphismes discontinus des algèbres de Banach commutatives séparables, Studia Math. 66 (1979), 119-141.

4. An algebra norm on $l^{2}$ whose restriction to $l^{1}$ is the usual $l^{1}$-norm, Proc. Amer. Math. Soc. 69 (1978), 303-307.

5. N. Jacobson, Lectures in abstract algebra, Vol. II, Van Nostrand, Princeton, N.J., 1953.

6. __ Structure of rings, Amer. Math. Soc. Colloq. Publ., vol. 37, Amer. Math. Soc., Providence, R.I., 1964.

SCHOOL OF MATHEMATICS, UNIVERSITY OF LEEDS, LEEDS LS2 9JT, ENGLAND 